\title{
Design of Energy Efficient Receiver and Transmitter for Optical Communication
}

\author{
Shivani Madhok ${ }^{1}$, Bishwajeet Pandey ${ }^{1}$, D M Akbar Hussain ${ }^{2}$, Bhagwan Das ${ }^{3}$ \\ and M F L Abdullah ${ }^{3}$ \\ ${ }^{1}$ Gyancity Research Lab and Jammu, India \\ shivanimadhok0@gmail.com,gyancity@gyancity.com \\ ${ }^{2}$ Aalborg University, Esbjerg, Denmark \\ akh@et.aau.dk \\ ${ }^{3}$ UTHM, Malaysia \\ he130092@siswa.uthm.edu.my,faiz@uthm.edu.my
}

\begin{abstract}
In the past, the modes of communication were wired, wireless and then came optical fiber communication into scenario. Nowadays, a new technology have been introduced known as Free Space Optics (FSO) that uses line of sight technology which uses lasers and photo detectors to provide optical connection. In this paper, the main aim of the author is to design an energy efficient transceiver for wireless optical communication. This paper consists of a transceiver design that is implemented on Xilinx ISE Design Suite 14.2 and results were tested on 28nm FPGA platform that is Kintex-7. Our design is based on 28nm FPGA and the device used is XC7K160T, package used is FBG676 and it is working on -3 speed grade. The advantages of FSO over fibre are it provides unlimited bandwidth, freedom from licensing, speed is very high, and high bit rate. Nowadays, bit rate is $2.5 \mathrm{Gbps}$ and in future it is expected to be 10Gbps. In this paper frequency scaling technique is used and frequency is varied to obtain power consumption of the Transceiver. Airflow has been kept 250 LFM and medium Heat sink. Power has been analyzed at different temperature range. At the end it can conclude that the maximum power consumption is at $2.2 \mathrm{GHz}$ frequency and minimum power consumption is at 1.2 $\mathrm{GHz}$. In terms of temperature maximum power is consumed at $56.7 \mathrm{oC}$ and minimum is consumed at $210 \mathrm{C}$.
\end{abstract}

Keywords: Energy Efficient, Free Space Optical Communication, FPGA, Transmitter, Receiver

\section{Introduction}

Communication requires any media to exchange information. Earlier the modes of communication were wired, wireless and then came optical fiber communication into scenario. Nowadays, a new technology have been introduced known as Free Space Optics (FSO) that uses line of sight technology which uses lasers and photo detectors to provide optical connection. Bit rate is very high that's why it is in demand these days. The approximate value of bit rate is $2.5 \mathrm{Gbps}$. Nowadays dual communication is in new trend and many corporations are using this technology to enhance its capability [1]. Dual communication involves both wired as well as wireless communication [1]. Wireless communication is one of the medium of communication that involves transfer of information without using electrical signals. Wireless communication can also be called as "Unguided Media". Other technologies are also emerging day-by-day OpticalWireless known as "FiWi" access network is a newly emerged access network

Received (July 26, 2017), Review Result (November 15, 2017), Accepted (November 20, 2017) 
architecture that uses passive optical networks (PONs) with wireless mesh networks (WMNs) to provide low cost, high bandwidth Internet access [2]. Wireless Optical communication technology is being used to design an eco-system that comprises of wireless communication, navigation, natural user interfaces and many more [3]. Other technology that is underwater optical wireless communication uses visible light for communication purpose because of the high data rate compared to other technologies and has low path attenuation compared to radio frequency (RF) technology [4]. In this paper, the main aim of the author is to design an energy efficient [11-14] transceiver for wireless optical communication. Figure 1 represents the different modes of communication. Earlier wired communication was in trend and now due to users demand for high bit rate, low error rate and high speed wireless optical communication (FSO) is preferred over all.

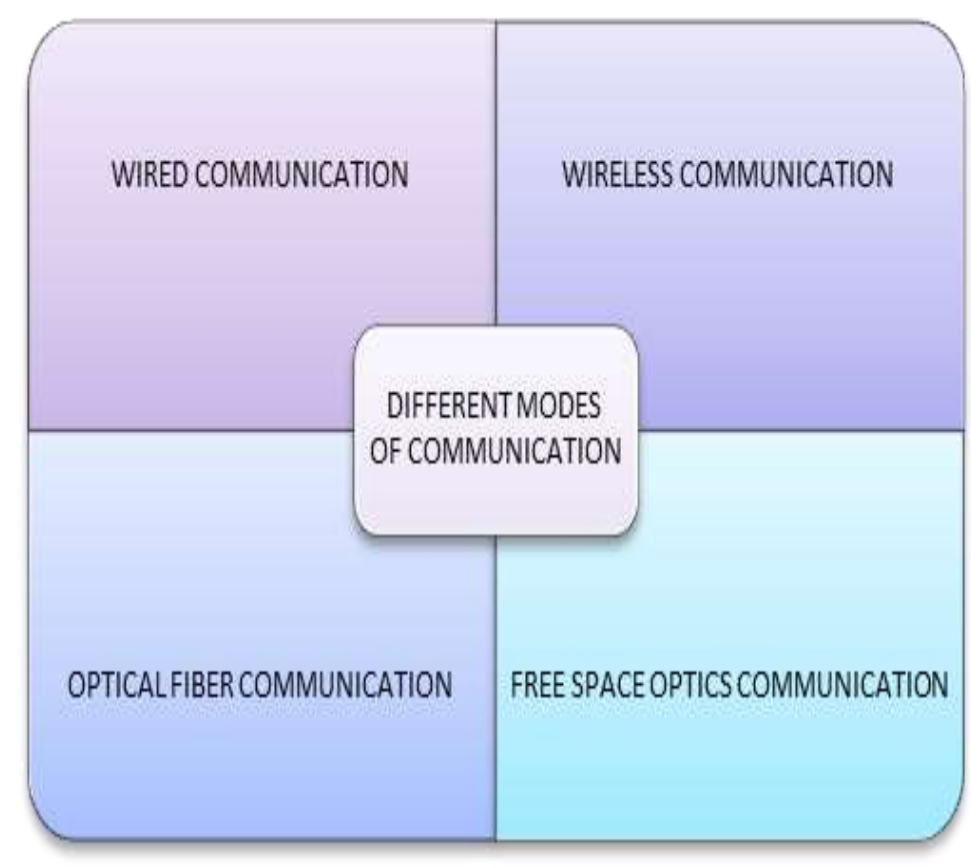

Figure 1. Different Modes of Communication

This paper consists of a transceiver design that is implemented on Xilinx ISE Design Suite 14.2 and results were tested on 28nm FPGA platform that is Kintex-7. Our design is based on 28nm FPGA and the device used is XC7K160T, package used is FBG676 and it is working on -3 speed grade. Table 1 shows different parameters in kintex-7 FPGA. Our main aim of this paper is to design a low power design for this we have taken different frequencies and calculated its power. Today's demand forces us to design the low power energy efficient device which takes lesser time for its execution.

Table 1. Different Parameters in Kintex-7 FPGA

\begin{tabular}{|l|l|}
\hline IO pins & 676 \\
\hline LUT Elements & 101400 \\
\hline Flip Flop & 202800 \\
\hline DSPS & 600 \\
\hline Available IOBS & 400 \\
\hline Gb transceiver & 8 \\
\hline Block RAM & 325 \\
\hline GTXE2 Transceiver & 8 \\
\hline PCI Buses & 1.1 \\
\hline
\end{tabular}




\begin{tabular}{|ll|l|}
\hline MMCMS & 8 \\
\hline Min operating temperature & 0 degree Celsius \\
\hline $\begin{array}{l}\text { Reference operating } \\
\text { temperature }\end{array}$ & 85 degree Celsius \\
\hline $\begin{array}{l}\text { Maximum operating } \\
\text { Temperature }\end{array}$ & 85 degree Celsius \\
\hline $\begin{array}{l}\text { Minimum operating } \\
\text { voltage }\end{array}$ & $0.97 \mathrm{~V}$ \\
\hline $\begin{array}{l}\text { Reference operating } \\
\text { Voltage }\end{array}$ & $0.97 \mathrm{~V}$ \\
\hline $\begin{array}{l}\text { Maximum } \\
\text { Voltage }\end{array}$ & $1.03 \mathrm{~V}$ \\
\hline Temperature Grade Letter & $\mathrm{C}$ \\
\hline
\end{tabular}

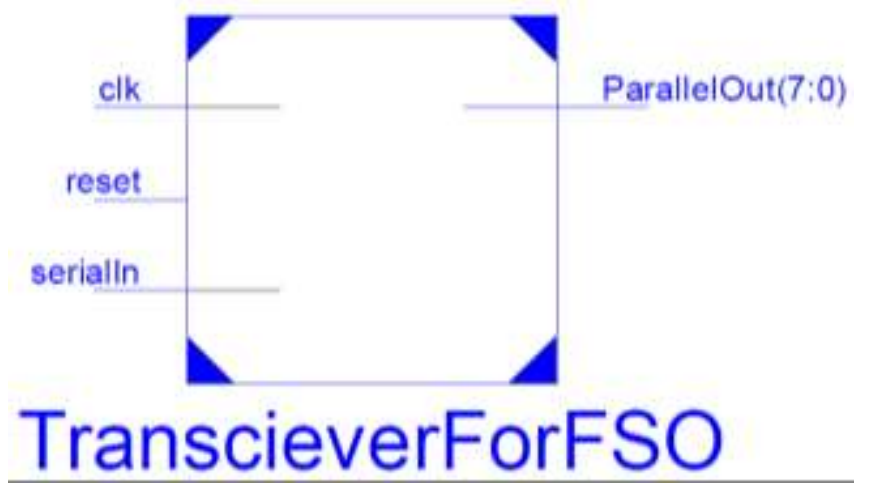

Figure 2. Symbol of Transceiver for FSO

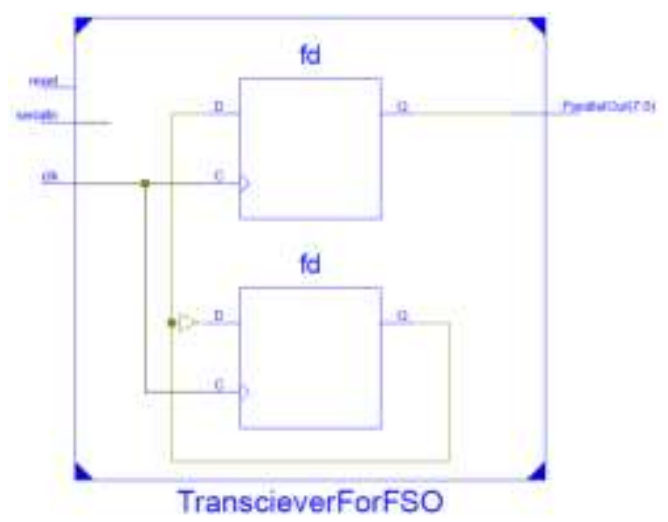

Figure 3. Schematic Diagram of Transceiver for FSO

Transceiver symbol as shown in Figure 2 consists of three inputs and one output. The design is clocked and works according to the clock input. The data or signal is given to the transceiver using serialIn and in taken out using parallelOut. Figure 3 shows the schematic diagram of transceiver for Free Space Optics (FSO). Free Space Optics (FSO) is a line of sight technology that does not use fibres but uses lasers and photo detectors to provide optical connections between the 2 points for communication purpose. Nowadays Free Space Optics (FSO) has become a high bandwidth alternative to fibre optic cabling [4]. The advantages of FSO over fibre are it provides unlimited bandwidth, freedom from licensing, speed is very high, and high bit rate. Nowadays, bit rate is $2.5 \mathrm{Gbps}$ and in future it is expected to be 10Gbps. The disadvantage of FSO over fibre is that laser power attenuation through the atmosphere, beam dispersion occurs, fog and rain affects the 
radiations. FSO is also called "Free Space Photonics". Free space here means air, outer space and vacuum. The only essential requirement for Free Space Optics (FSO) or optical wireless transmission is line of sight between the two ends of the link. This technology uses two telescopes for transmission and reception. Light beams travels from one telescope to another using low power infrared lasers. Receivers are telescopic lenses that are able to collect the photon stream coming from the transmitter. Figure 4 shows the mechanism of free space optical communication. This technology is gaining popularity because it can function over distance of several kilometres. Free space optics is much more secure than other modes of communication as it cannot be detected by spectrum analyser or RF meters and interception is also very difficult.

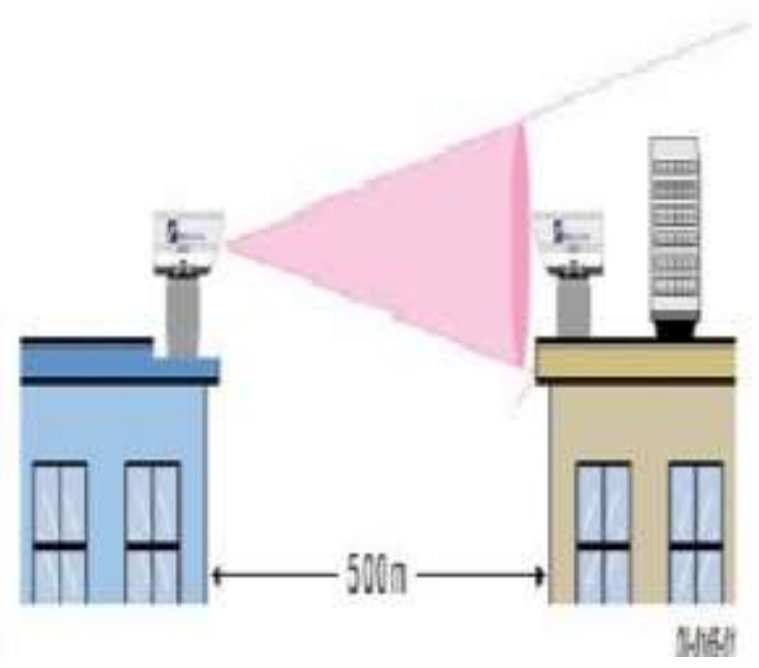

Figure 4. Free Space Optics Mechanism

Table 2. Set of Frequencies Taken in Consideration

\begin{tabular}{|l|l|}
\hline Frequency & Mobile Set \\
\hline $1400 \mathrm{MHz}$ & Nokia Lumia 710 \\
\hline $1.2 \mathrm{GHz}$ & Samsung Galaxy Core \\
\hline $2100 \mathrm{MHz}$ & I phone6 \\
\hline $1700 \mathrm{MHz}$ & HTC/T \\
\hline $1800 \mathrm{MHz}$ & Micromax X091 \\
\hline $2.2 \mathrm{GHz}$ & Sony Xperia Z1 \\
\hline
\end{tabular}

In this paper frequency scaling technique is used and frequency is varied to obtain power consumption of the Transceiver. The frequencies taken under consideration are shown in Table 2. Different sets of mobile phones operate at different frequencies and these frequencies are considered for designing an energy efficient device. There are many other types of techniques like capacitance scaling technique [5], thermal scaling [6], clock gating [7], various design goals [8], impedance matching with different logic family, scalable implementation scheme [9] and mapping. Airflow and heat sink are main parameters while analyzing the thermal dissipation in the circuit [10]. In this work we have taken constant value of air flow and heat sink. Airflow has been kept 250 LFM and medium Heat sink. Power has been analyzed at different temperature range. Four different regions have been considered having different temperature like Furnace Creek Ranch $(329.85 \mathrm{~K})$, Mohenjo-Daro $(326.65 \mathrm{~K})$, median temperature of Delhi $(313.15 \mathrm{~K})$ and standard normal temperature (294.15K). 


\section{POWER ANALYSIS}

\subsection{Power Analysis at 56.7 degree Celsius}

Table 3. Power Analysis at $56.7^{\circ} \mathrm{Celsius}$

\begin{tabular}{|l|l|}
\hline Frequency & Total Power Dissipated \\
\hline $1400 \mathrm{MHz}$ & 0.159 \\
\hline $1.2 \mathrm{GHz}$ & 0.152 \\
\hline $2100 \mathrm{MHz}$ & 0.186 \\
\hline $1700 \mathrm{MHz}$ & 0.171 \\
\hline $1800 \mathrm{MHz}$ & 0.174 \\
\hline $2.2 \mathrm{GHz}$ & 0.188 \\
\hline
\end{tabular}

There is $19.14 \%$ saving in total power dissipation with $1.2 \mathrm{GHz}$ when compared with 2.2 GHz at 56.7 Degree Celsius as shown in Figure 5 and Table 3 .

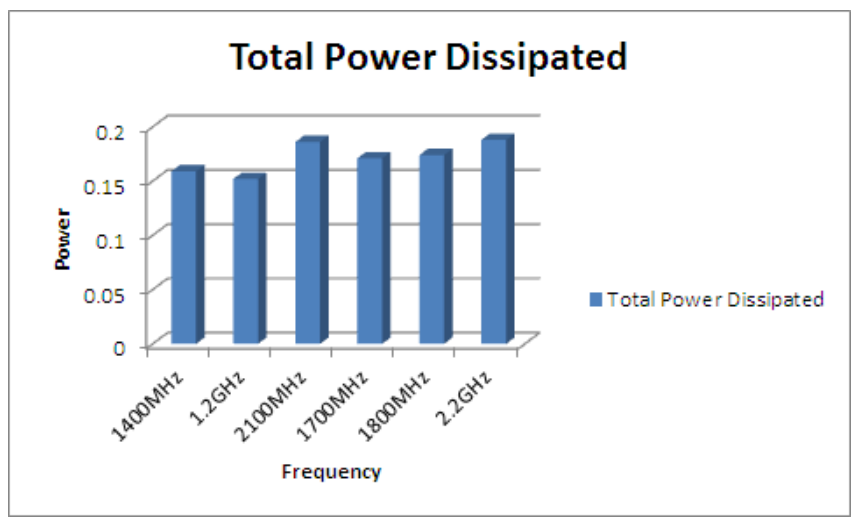

Figure 5. Power Dissipation at $56.7^{\circ} \mathrm{Celsius}$ Temperature

\subsection{Power Analysis at 53.5 Degree Celsius}

Table 4. Power Analysis at $53.5^{\circ}$ Celsius

\begin{tabular}{|l|l|}
\hline Frequency & Total Power Dissipated \\
\hline $1400 \mathrm{MHz}$ & 0.148 \\
\hline $1.2 \mathrm{GHz}$ & 0.141 \\
\hline $2100 \mathrm{MHz}$ & 0.175 \\
\hline $1700 \mathrm{MHz}$ & 0.160 \\
\hline $1800 \mathrm{MHz}$ & 0.163 \\
\hline $2.2 \mathrm{GHz}$ & 0.177 \\
\hline
\end{tabular}

There is $20.33 \%$ saving in total power dissipation with $1.2 \mathrm{GHz}$ when compared with 2.2 GHz at 56.7 Degree Celsius as shown in Figure 6 and Table 4 . 


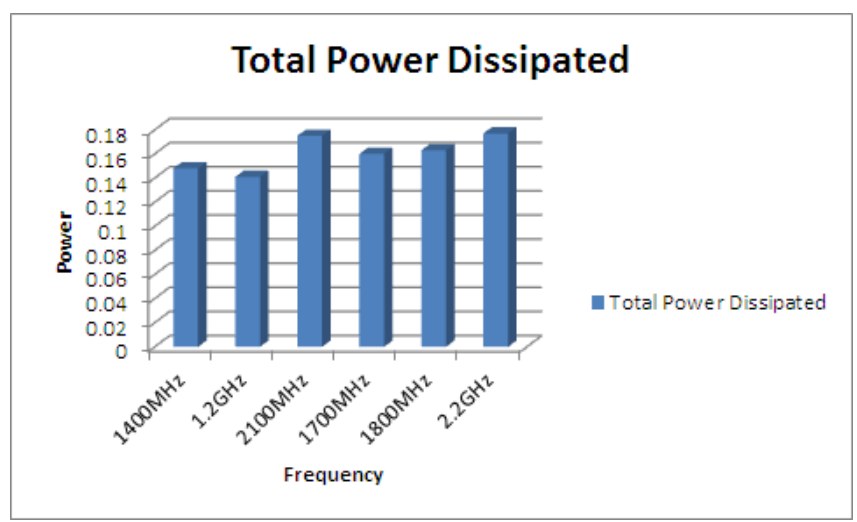

Figure 6. Power Dissipation at $53.5^{\circ} \mathrm{Celsius}$ Temperature

\subsection{Power Analysis at 40 Degree Celsius}

Table 5. Power Analysis at $40^{\circ} \mathrm{Celsius}$

\begin{tabular}{|l|l|}
\hline Frequency & Total Power Dissipated \\
\hline $1400 \mathrm{MHz}$ & 0.114 \\
\hline $1.2 \mathrm{GHz}$ & 0.107 \\
\hline $2100 \mathrm{MHz}$ & 0.141 \\
\hline $1700 \mathrm{MHz}$ & 0.126 \\
\hline $1800 \mathrm{MHz}$ & 0.129 \\
\hline $2.2 \mathrm{GHz}$ & 0.143 \\
\hline
\end{tabular}

There is $25.17 \%$ saving in total power dissipation with $1.2 \mathrm{GHz}$ when compared with 2.2 GHz at 56.7 Degree Celsius as shown in Figure 7 and Table 5.

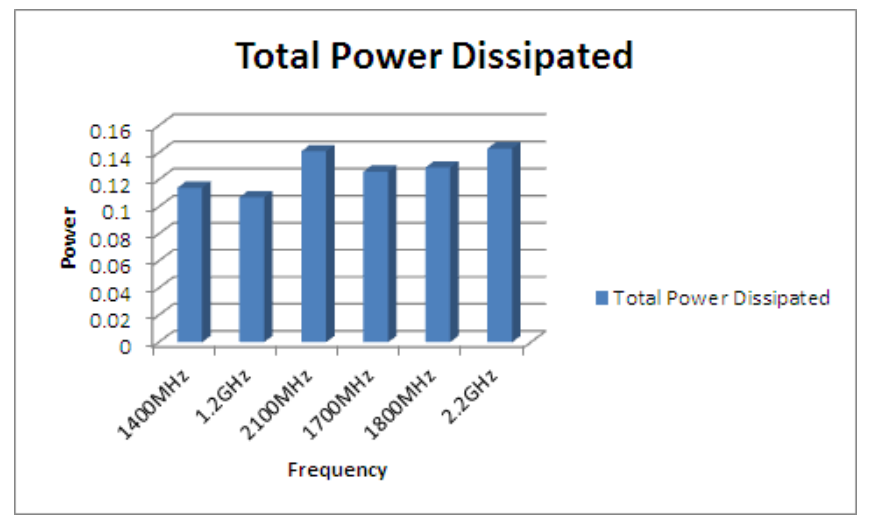

Figure 7. Power Dissipation at $40^{\circ}$ Celsius Temperature 


\subsection{Power Analysis at 21 Degree Celsius}

Table 6. Power Analysis at $21^{\circ} \mathrm{Celsius}$

\begin{tabular}{|l|l|}
\hline Frequency & Total Power Dissipated \\
\hline $1400 \mathrm{MHz}$ & 0.090 \\
\hline $1.2 \mathrm{GHz}$ & 0.083 \\
\hline $2100 \mathrm{MHz}$ & 0.118 \\
\hline $1700 \mathrm{MHz}$ & 0.102 \\
\hline $1800 \mathrm{MHz}$ & 0.105 \\
\hline $2.2 \mathrm{GHz}$ & 0.119 \\
\hline
\end{tabular}

There is $30.25 \%$ saving in total power dissipation with $1.2 \mathrm{GHz}$ when compared with 2.2 GHz at 56.7 Degree Celsius as shown in Figure 8 and Table 6.

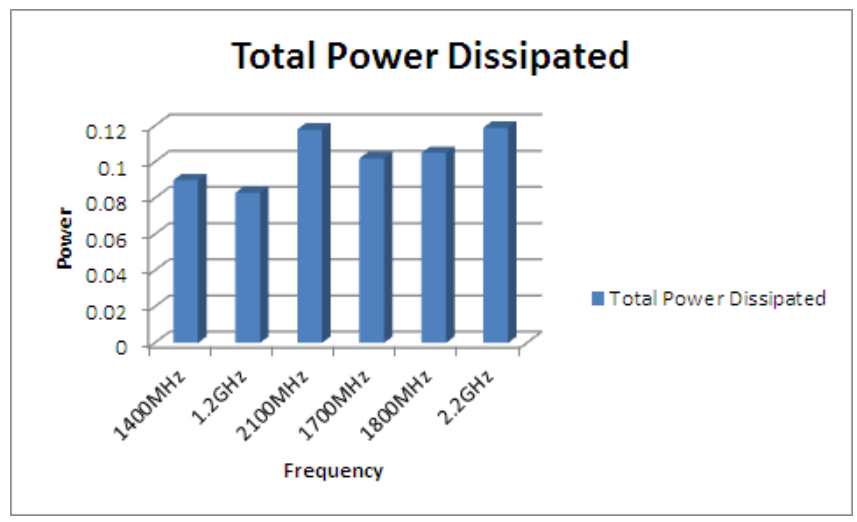

Figure 8. Power Dissipation at $21^{\circ} \mathrm{Celsius}$ Temperature

\subsection{Power Consumption at Different Frequencies at Different Temperatures}

Table 7. IO Power Analysis for Different Frequencies at Different Temperatures

\begin{tabular}{|l|l|l|l|l|}
\hline Frequency & $\mathbf{5 6 . 7}^{\mathbf{}} \mathbf{C}$ & $\mathbf{5 3 . 5}^{\mathbf{C}}$ & $\mathbf{4 0}^{\mathbf{}} \mathbf{C}$ & $\mathbf{2 1}^{\mathbf{}} \mathbf{C}$ \\
\hline $1400 \mathrm{MHz}$ & 0.159 & 0.148 & 0.114 & 0.090 \\
\hline $1.2 \mathrm{GHz}$ & 0.152 & 0.141 & 0.107 & 0.083 \\
\hline $2100 \mathrm{MHz}$ & 0.186 & 0.175 & 0.141 & 0.118 \\
\hline $1700 \mathrm{MHz}$ & 0.171 & 0.160 & 0.126 & 0.102 \\
\hline $1800 \mathrm{MHz}$ & 0.174 & 0.163 & 0.129 & 0.105 \\
\hline $2.2 \mathrm{GHz}$ & 0.188 & 0.177 & 0.143 & 0.119 \\
\hline
\end{tabular}

From Table 7 and Figure 9 it can conclude that the maximum power consumption is at $2.2 \mathrm{GHz}$ frequency and minimum power consumption is at $1.2 \mathrm{GHz}$. In terms of temperature maximum power is consumed at $56.7^{\circ} \mathrm{C}$ and minimum is consumed at $21^{\circ} \mathrm{C}$. 


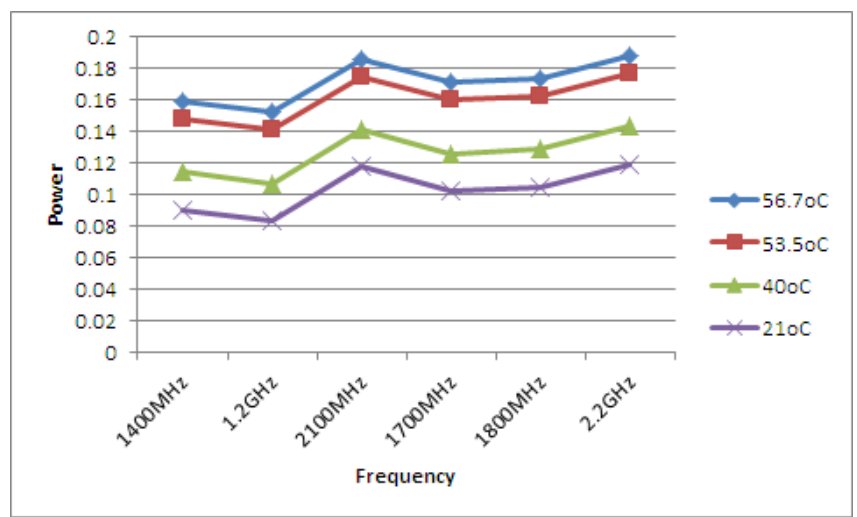

\section{Figure 9. IO Power Analysis for Different Frequencies at Different Temperatures}

\section{Conclusion}

The design is low power energy efficient and the code has been implemented in Xilinx ISE Design Suite 14.2 and results were tested on 28nm FPGA platform. This transceiver design consists of three inputs and one output. The device has been clocked and tested at different frequency levels at different temperature ranges. In this paper thermal analysis has been done to make our device energy efficient device. Four different values of temperature of four different regions have been considered like Furnace Creek Ranch $(329.85 \mathrm{~K})$, Mohenjo-Daro (326.65K), median temperature of Delhi $(313.15 \mathrm{~K})$ and standard normal temperature $(294.15 \mathrm{~K})$. At the end it can conclude that the maximum power consumption is at $2.2 \mathrm{GHz}$ frequency and minimum power consumption is at 1.2 $\mathrm{GHz}$. In terms of temperature maximum power is consumed at $56.7 \mathrm{oC}$ and minimum is consumed at $210 \mathrm{C}$.

\section{Future Scope}

The future scope of "Energy Efficient Receiver and Transmitter Design for Free Space Optical Communication" on 28nm FPGA is that we can also implement this design on $22 \mathrm{~nm}$ or $18 \mathrm{~nm}$ FPGA. The FPGA family used in this paper is Kintex7. Different FPGA families like automotive Artix7, automotive Coolrunner2, automotive Spartan, automotive Spartan-3A DSP, automotive Spartan 3A, automotive Spartan 3E, automotive Spartan6, Spartan3, Spartan3E can also be used. Here, Thermal Aware and frequency scaling technique has been implemented by varying the value of temperature at different frequency. The design can be redesigned with other energy efficient technique like capacitance scaling, clock gating, and impedance matching with different logic family, and mapping. The analysis in this paper is done only at $1400 \mathrm{MHz}, 1.2 \mathrm{GHz}, 1700 \mathrm{MHz}$, $1800 \mathrm{MHz}, 2100 \mathrm{MHz}$ and $2.2 \mathrm{GHz}$. Frequency range can be varied to make the design more energy efficient. IO Standards can be introduced to enhance the capability of the device or to make it more efficient. IO standards like LVCMOS, SSTL, HSTL MOBILE_DDR and many more can be used.

\section{References}

[1] K. Ishio, "Development of a dual communication system LSI", Consumer Electronics (GCCE), 2012 IEEE 1st Global Conference on. IEEE, (2012).

[2] S. Zhou, "Distributed wireless communication system: a new architecture for future public wireless access", Communications Magazine, IEEE, vol. 41, no 3, (2003), pp. 108-113.

[3] G. S. Wilson, "Free-Space Optical MIMO Transmission With< img src="/images/tex/228. gif" alt=" Q">-ary PPM", Communications, IEEE Transactions on, vol. 53, no 8, (2005), pp. 1402-1412. 
[4] I. I. Kim and J. K. Eric, "Availability of free-space optics (FSO) and hybrid FSO/RF systems", ITCom 2001: International Symposium on the Convergence of IT and Communications. International Society for Optics and Photonics, (2001).

[5] B. Pandey, "Capacitance scaling based energy efficient FIR filter for digital signal processing", International Conference on Optimization, Reliabilty, and Information Technology (ICROIT). IEEE, (2014).

[6] K. Fushinobu and H. Tomoyuki, "Thermal scaling consideration of Si MOSFETs with gate length typically larger than $100 \mathrm{~nm}$ ", 27th Annual Semiconductor Thermal Measurement and Management Symposium (SEMI-THERM), IEEE, (2011).

[7] Y. Zhang, "Automatic Register Transfer level CAD tool design for advanced clock gating and low power schemes", International SoC Design Conference (ISOCC). IEEE, (2012).

[8] Z. Liu and W. Zhenxing, "Analysis to Goal-Driven Design".

[9] P.C. Wu, L.G. Chen and T.D. Chiueh, "Scalable implementation scheme for multirate FIR filters and its application in efficient design of subband filter banks", IEEE transactions on circuits and systems for video technology, vol. 6, no. 4, (1996), pp. 407-410.

[10] T. Kumar, "Simulation of voltage based efficient fire sensor on FPGA using SSTL IO standards", International Conference on Robotics and Emerging Allied Technologies in Engineering (iCREATE). IEEE, (2014).

[11] T. Kumar, B. Pandey, S.H.A. Musavi and N. Zaman, "CTHS Based Energy Efficient Thermal Aware Image ALU Design on FPGA", Springer Wireless Personal Communications, An International Journal, vol. 83 , no. 1 , (2015).

[12] D. Singh, K. Garg, R. Singh, B. Pandey, K. Kalia and H. Noori, "Thermal aware Internet of Things Enable Energy Efficient Encoder Design for security on FPGA", International Journal of Security and Its Applications, http://www.sersc.org/journals/IJSIA/vol9_no6_2015/26.pdf, vol. 9, no 6, (2015), pp. 271-278

[13] , S.H.A, Musavi, B.S. Chowdhry, T. Kumar, B. Pandey and W. Kumar, "IoTs Enable Active Contour Modeling Based Energy Efficient and Thermal Aware Object Tracking on FPGA", Springer Wireless Personal Communications, vol. 82, no. 20, (2015), pp. 1-15.

[14] B. S. Chowdhry, B. Pandey, T. Kumar and T. Das, "Mobile DDR IO Standard Based High Performance Energy Efficient Portable ALU Design on FPGA", Springer Wireless Personal Communications, An International Journal, vol. 76, no. 3, (2014), pp. 569-578. 
International Journal of Signal Processing, Image Processing and Pattern Recognition Vol. 10, No. 11 (2017) 